\title{
Postmenopausal breast cancer and oestrogen associations with the IgA-coated and IgA-noncoated faecal microbiota
}

James J Goedert ${ }^{\star}, 1$, Xing Hua ${ }^{1}$, Agata Bielecka ${ }^{2}$, Isao Okayasu ${ }^{3}$, Ginger L Milne ${ }^{4}$, Gieira S Jones ${ }^{1}$, Mutsunori Fujiwara ${ }^{5}$, Rashmi Sinha ${ }^{1}$, Yunhu Wan ${ }^{1}$, Xia $\mathrm{Xu}^{6}{ }^{6}$, Jacques Ravel ${ }^{7}$, Jianxin Shi ${ }^{1}$, Noah W Palm ${ }^{2}$ and Heather Spencer Feigelson ${ }^{8}$

\begin{abstract}
${ }^{1}$ Division of Cancer Epidemiology and Genetics, National Cancer Institute, 9609 Medical Center Drive, Room 6E106, MSC 9767, Bethesda, MD 20892, USA; ${ }^{2}$ Department of Immunobiology, Yale University School of Medicine, New Haven, CT 21702, USA; ${ }^{3}$ Department of Pathology, Kitasato University School of Medicine, Sagamihara, Kanagawa 252-0374, Japan; ${ }^{4}$ Division of Clinical Pharmacology, Department of Medicine, Vanderbilt University Medical Center, Nashville, TN 37232, USA; ${ }^{5}$ Department of Clinical Pathology, Japanese Red Cross Medical Center, Shibuya-ku, Tokyo 150-0012, Japan; ${ }^{6}$ Cancer Research Technology Program, Leidos Biomedical Research Inc., Frederick National Laboratory for Cancer Research, Frederick, MD 21702, USA; ${ }^{7}$ University of Maryland Medical School, Baltimore, MD 21201, USA and ${ }^{8}$ Institute for Health Research, Kaiser Permanente Colorado, Denver, CO 80237, USA
\end{abstract}

Background: The diversity and composition of the gut microbiota may affect breast cancer risk by modulating systemic levels of oestrogens and inflammation. The current investigation tested this hypothesis in postmenopausal women by identifying breast cancer associations with an inflammation marker, oestrogen levels, and faecal microbes that were or were not coated with mucosal immunoglobulin A ( $(g A)$.

Methods: In this population-based study, we compared 48 postmenopausal breast cancer cases (75\% stage 0-1, 88\% oestrogenreceptor positive) to 48 contemporaneous, postmenopausal, normal-mammogram, age-matched controls. Microbiota metrics employed 16S rRNA gene amplicon sequencing from IgA-coated and -noncoated faecal microbes. High-performance liquid chromatography/mass spectrometry (HPLC/MS) and radioimmunoassay were used to quantify urine prostaglandin E metabolite (PGE-M), a possible marker of inflammation; urine oestrogens and oestrogen metabolites were quantified by HPLC/MS-MS.

Results: Women with pre-treatment breast cancer had non-significantly elevated oestrogen levels; controls' (but not cases') oestrogens were directly correlated with their IgA-negative microbiota alpha diversity $(P=0.012)$. Prostaglandin $E$ metabolite levels were not associated with case status, oestrogen levels, or alpha diversity. Adjusted for oestrogens and other variables, cases had significantly reduced alpha diversity and altered composition of both their IgA-positive and IgA-negative faecal microbiota. Cases' faecal microbial IgA-positive imputed Immune System Diseases metabolic pathway genes were increased; also, cases' IgApositive and IgA-negative imputed Genetic Information Processing pathway genes were decreased $(P \leqslant 0.01)$.

Conclusions: Compared to controls, breast cancer cases had significant oestrogen-independent associations with the IgA-positive and IgA-negative gut microbiota. These suggest that the gut microbiota may influence breast cancer risk by altered metabolism, oestrogen recycling, and immune pressure.

*Correspondence: Dr JJ Goedert; E-mail: goedertj@mail.nih.gov

Received 20 July 2017; revised 7 November 2017; accepted 8 November 2017; published online 23 January 2018

(C) 2018 Cancer Research UK. All rights reserved 0007 - 0920/18 
In postmenopausal women, breast cancer risk is elevated with physical inactivity, weight gain, adiposity, and subclinical obesityrelated inflammation (Hong et al, 2013; Gunter et al, 2015; Tamimi et al, 2016). The cancer risk due to these factors could be mediated, in part, by higher levels of endogenous oestrogens (Brown and Simpson, 2012), which are predictive of breast cancer risk (Key et al, 2002, 2003; Sampson et al, 2017), or with alterations of host inflammation and metabolism.

A postulated underlying mechanism for breast cancer is inflammation-related upregulation of cyclooxygenase 2 (COX2) and its product, prostaglandin $\mathrm{E}_{2}$ (PGE), which results in increased aromatase expression in adipose tissue and increased conversion of androgen precursors to oestrogens (Subbaramaiah et al, 2012; Bowers et al, 2015). Systemically, epidemiologic and clinical studies have found that inhibition of the COX2-PGE pathway through use of aspirin, and to a lesser extent, other nonsteroidal anti-inflammatory drugs is associated with a $10-20 \%$ reduced risk of oestrogen receptor (ER)-positive breast cancer incidence or recurrence (Gierach et al, 2008; Bardia et al, 2011; Silva et al, 2012; Bowers et al, 2014; de Pedro et al, 2015; Yiannakopoulou, 2015; Zhong et al, 2015). Notably, high urinary levels of the major prostaglandin $\mathrm{E}_{2}$ metabolite (PGE-M) have been predictive of increased risk of breast cancer in three prospective studies of postmenopausal women, albeit with some inconsistencies (Kim et al, 2013, 2017; Cui et al, 2014).

Breast cancer associations with obesity, inactivity, inflammation, and oestrogen levels could also reflect differences among individuals in their gut microbial communities (i.e., their intestinal microbiota) (Plottel and Blaser, 2011; Boulangé et al, 2016; Kwa et $a l, 2016)$. The pioneering studies of Adlercreutz and his colleagues, starting nearly 50 years ago, demonstrated with exquisite clarity the fundamental role of the gut microbiota in maintaining systemic oestrogen homeostasis through enterohepatic circulation (Tikkanen et al, 1973; Adlercreutz and Martin, 1980). Based on this foundation, we showed that a higher level of urinary oestrogens or altered oestrogen metabolism was associated with reduced diversity and altered composition of the faecal microbiota in generally healthy men and postmenopausal women (Flores et al, 2012a; Fuhrman et al, 2014). Moreover, we recently reported statistically significant differences in the diversity and composition of the faecal microbiota in postmenopausal women with newly diagnosed, pre-treatment breast cancer compared to closely matched control women (Goedert et al, 2015). The cancer association with diversity of the faecal microbiota was independent of higher oestrogen levels in the cases (Goedert et al, 2015).

A potential link between breast cancer's associations with inflammation and the gut microbiota is secretory immunoglobulin A (IgA), which is produced by plasma cells resident in the gut mucosa and which plays an essential role in maintaining the integrity of the mucosal barrier by recognising and regulating the composition of the gut microbial community. This critical function of mucosal secretory IgA has been well described for two serious human conditions - severe malnutrition (Kwashiorkor) in African infants and inflammatory bowel disease in American adults functions that have been recapitulated in murine models of these conditions (Palm et al, 2014; Kau et al, 2015). To evaluate secretory IgA and the microbiota, the general approach is to distinguish, physically separate, and characterise IgA-coated from IgA-noncoated bacteria based on staining the mixed microbial population with anti-IgA.

The current investigation sought to identify postmenopausal breast cancer associations with urinary levels of oestrogens and oestrogen metabolites (EMs), with the inflammation marker PGE$\mathrm{M}$, and particularly with the participants' IgA-positive and IgAnegative faecal microbiota.

\section{MATERIALS AND METHODS}

Participant recruitment and specimen collection and handling. Following review and approval by institutional review boards at Kaiser Permanente in Colorado (KPCO) and the National Cancer Institute, we recruited female KPCO members ages 50-74 years. Cases were women found to have suspicious lesions on screening mammography that proved to be breast cancer on biopsy. Following informed consent and recruitment, participants provided specimens and data prior to definitive surgical or systemic therapy. Controls were women with normal screening mammograms, contemporaneous with and frequency matched by age to the cases and recruited with the same methods. Each participating woman provided questionnaire data (used to confirm menopausal status as well as ascertain breast cancer risk factors), urine without preservative, and contemporaneous faeces collected (typically early or mid-morning) in phosphate-buffered saline (PBS) that was frozen at home at $-20^{\circ} \mathrm{C}$, transported on dry ice within days by KPCO staff, and thereafter maintained at or below $-80^{\circ} \mathrm{C}$ until use. For both cases and controls, exclusionary conditions were any history of previous cancer (except non-melanoma skin cancer), inflammatory bowel disease, diverticulitis, or bariatric surgery; other gastrointestinal surgery within 6 months; any antibiotic prescription within 6 months; and any oestrogenic or other hormone prescription within 12 months. Additional details are provided elsewhere (Feigelson et al, 2014; Goedert et al, 2015).

IgA-Seq and 16S rRNA gene sequencing. Faecal samples, plus quality control (QC) samples (nine water, 12 Chemostat-A replicates, 12 Chemostat-B replicates (Santiago-Rodriguez et al, 2015)), were thawed on ice for $\sim 30 \mathrm{~min}$ before aliquoting $\sim 100 \mathrm{mg}$ of faeces into $2 \mathrm{ml}$ screwcap tubes (Sarstedt, Nümbrecht, Germany). Faecal aliquots were diluted in $1 \mathrm{ml}$ PBS per $100 \mathrm{mg}$ faeces, and homogenised by adding ceramic beads to each tube (Lysing Matrix D, MP Biomedicals) followed by bead beating for $7 \mathrm{~s}$ (Biospec Mini-Beadbeater). Homogenised faecal samples were centrifuged at $50 \mathrm{~g}$ at $4{ }^{\circ} \mathrm{C}$ for $15 \mathrm{~min}$ to pellet large particles, and $50 \mu \mathrm{l}$ of supernatant containing bacteria was collected into a new microfuge tube. Samples were washed three times to remove free IgA by resuspending in $1 \mathrm{ml}$ PBS containing $1 \%$ bovine serum albumin (PBS/BSA) and pelleting at $8000 \mathrm{~g}$ at $4{ }^{\circ} \mathrm{C}$. At this stage, a $20 \mu \mathrm{l}$ aliquot of washed bacteria was saved for 16S rRNA gene amplification and sequencing as the 'unsorted' microbiota. After three washes, bacteria were resuspended in $50 \mu \mathrm{l}$ of PBS/BSA containing 20\% Normal Mouse Serum (Jackson Immunochemicals, West Grove, PA, USA). After 15 min on ice, an equal volume of PE-Anti-Human IgA $(1: 12.5$; Miltenyi Biotec, Bergisch Gladbach, Germany) was added, and samples were incubated on ice for an additional $30 \mathrm{~min}$. The IgA-treated microbiota were washed three times with PBS/BSA before cell sorting. At least 500000 IgA-positive and IgA-negative bacterial cells per specimen were isolated via cell sorting (FACSAria, BD Biosciences, San Jose, CA, USA). After sorting, the specimens were pelleted and frozen at $-80^{\circ} \mathrm{C}$.

DNA was isolated from the unsorted, IgA-positive and IgAnegative microbiota with the PowerLyzer UltraClean Microbial DNA Isolation Kit (MoBio/Qiagen, Carlsbad, CA, USA) following the manufacturers' instructions. The V4 region of the 16S rRNA gene was amplified via PCR (32 cycles) using primers containing dual indices and sequenced on a MiSeq ( 2 x 250 Paired-end; Illumina) as previously described (https://www.ncbi.nlm.nih.gov/ pmc/articles/PMC3753973/).

Detection and quantification of oestrogens and oestrogen metabolites in urine. As reported previously (Fuhrman et al, 2014; Goedert et al, 2015), parent oestrogens (oestrone and estradiol) and 13 EMs were quantified in urine by liquid 
chromatography/tandem mass spectrometry. Briefly, LC-MS/MS analysis was performed using a Thermo TSQ Quantum Ultra triple quadrupole mass spectrometer (Thermo Scientific, San Jose, CA, USA) coupled with a Prominence UFLC system (Shimadzu Scientific Instruments, Columbia, MD, USA). Both LC and mass spectrometer were controlled by Xcalibur software (Thermo Scientific). Twelve stable isotopically labelled oestrogens and EMs were used to account for losses during sample preparation and assays, which included deuterated estriol, (C/D/N Isotopes, Inc., Pointe-Claire, QC, Canada); deuterated 16-epiestriol (Medical Isotopes, Inc., Pelham, NH, USA); and 13C-labelled oestrone, estradiol, 2-hydroxyestrone, 2-methoxyestrone, 2-hydroxyestradiol, 2-methoxyestradiol, 2-hydroxyestrone-3-methyl ether, 4hydroxyestrone, 4-methoxyestrone, and 4-methoxyestradiol (Cambridge Isotope Laboratories, Andover, MA, USA). To standardise the values, creatinine was measured in the urine specimens by the Clinical Chemistry laboratory at the NIH Clinical Center.

Detection and quantification of the major urinary metabolite of $\mathrm{PGE}_{2}$ (PGE-M) in urine. In urine aliquots, matching those used for detection of oestrogens and EMs, the major urinary metabolite of PGE (9,15-dioxo-11 $\alpha$-hydroxy-13,14-dihydro-2,3,4,5-tetranorprostane-1,20-dioic acid, PGE-M) was detected and quantified using two different techniques (radioimmunoassay and mass spectrometry) by two independent laboratories and standardised to the level of creatinine present in the urine sample.

Method 1 - radioimmunoassay. Prostaglandin E metabolite itself is unstable when stored at room temperature for more than $2 \mathrm{~h}$. When treated under basic conditions, PGE-M undergoes hydrolysis and subsequent cyclisation. Previously, we developed a simplified radioimmunoassay (RIA) to quantify this product in human urine using a specific rabbit antibody obtained by immunisation with chemically synthesised 19-carboxy-11-deoxy13,14-dihydro-15-dehydro-2,3,4,5,20-pentanor-11ß-16e-cycloprostaglandin E1 (the stable compound, bicyclic PGE-MUM) (Okayasu et al, 2014). The RIA kit was produced by the Institute of Isotopes Co., Ltd. (Budapest, Hungary) and distributed by TFB, Inc. (Toshima-ku, Tokyo, Japan). This surrogate biomarker for PGE-M was quantified in individual urine samples after being processed as described previously (Okayasu et al, 2014). Briefly, all samples $(50 \mu \mathrm{l})$ were kept at room temperature for $30 \mathrm{~min}$ after the addition of $100 \mu \mathrm{l}$ of $1 \mathrm{~N} \mathrm{NaOH}$, and neutralised with $100 \mu \mathrm{l}$ of $1 \mathrm{~N} \mathrm{HCl}$. They were then diluted $(25 \times)$ with $1000 \mu$ l of $50 \mathrm{~mm}$ phosphate buffer ( $\mathrm{pH} 7.4$ ) containing $0.1 \%$ gelatin and $0.1 \%$ sodium azide. The prepared samples were pipetted into assay tubes, $50 \mu \mathrm{l}$ each. Then, $100 \mu \mathrm{l}$ of ${ }^{125}$ I-bicyclic PGE-M $(680 \mathrm{~Bq}, \sim 37 \mathrm{pg})$ and subsequently $100 \mu \mathrm{l}$ of rabbit antibicyclic PGE-M were added to each tube. After incubation at room temperature for $2 \mathrm{~h}, 250 \mu \mathrm{l}$ of separating reagent was added to each tube and incubation was further continued for $15 \mathrm{~min}$ at room temperature. After centrifugation at $2000 \mathrm{~g}$ for $10 \mathrm{~min}$, the supernatant was removed by decantation. The radioactivity in the residues was counted with an Auto Well Gamma Counter ARC1000 (ALOKA, Tokyo, Japan).

Method 2 - liquid chromatography/mass spectrometry. Levels of urinary PGE-M were measured in the Eicosanoid Core Laboratory at Vanderbilt University Medical Center as previously described (Morris et al, 2013). Briefly, urine samples were removed from $-80{ }^{\circ} \mathrm{C}$ storage and allowed to thaw at room temperature. Immediately, $1 \mathrm{ml}$ of sample was acidified to $\mathrm{pH} 3$ with $1 \mathrm{moll}^{-1}$ $\mathrm{HCl}$, and endogenous PGE-M was then converted to the $\mathrm{O}$ methyloxime derivative by treatment with $0.5 \mathrm{ml}$ of $16 \%(\mathrm{w} / \mathrm{v})$ methyloxime $\mathrm{HCl}$ in $1.5 \mathrm{moll}^{-1}$ sodium acetate buffer $(\mathrm{pH} 5)$ to stabilise the molecule from degradation. Following the 1-h incubation, the derivatised PGE-M was diluted with $10 \mathrm{ml}$ water adjusted to $\mathrm{pH} 3$, and the aqueous sample was extracted using a
C-18 Sep-Pak (Waters Corp., Milford, MA, USA). PGE-M was then eluted from the Sep-Pak with $5 \mathrm{ml}$ ethyl acetate, and any residual aqueous material was removed from the eluate by aspiration. The $\left[{ }^{2} \mathrm{H}_{6}\right]-\mathrm{O}$-methyloxime PGE-M internal standard (IS, $6.2 \mathrm{ng}$ in $10 \mu \mathrm{l}$ ethanol) was then added, and the eluate plus IS was evaporated under a continuous stream of nitrogen at $37^{\circ} \mathrm{C}$. The dried residue was resuspended in $50 \mu \mathrm{l}$ 95:4.9:0.1 (v/v/v) $5 \mathrm{mmoll}^{-1}$ ammonium acetate:acetonitrile:acetic acid for analysis using LC/MS.

Operational taxonomic unit assignments, taxonomy and diversity estimates, and imputed metagenomics functional pathways. The revised version of the Divisive Amplicon Denoising Algorithm (DADA2) package (Callahan et al, 2016) and SILVA (ver123) was used to assign $16 \mathrm{~S}$ rRNA gene sequences to operational taxonomic units (OTUs), calculate their relative abundances, and estimate alpha- and beta-diversity metrics for the IgA-positive, IgAnegative, and unsorted microbiota. The sequence reads were rarified to 10000 sequences per sample without replacement for alpha diversity calculations. Alpha diversity was estimated as follows. Richness: number of unique species-level OTUs, unadjusted for their relative abundances. Chaol: richness, but biascorrected for rare (singleton, doubleton) taxa. Phylogenetic diversity (PD)_whole_tree: sum of the branch lengths of a phylogenetic tree constructed from all taxa in a sample. Shannon index: a conservative estimate that adjusts for relative abundance of each taxon and that is defined as (negative) the sum over taxa of the product of the relative abundance of each taxon times the natural logarithm of its relative abundance.

To impute the relative abundances of microbial genes in the IgA-positive, IgA-negative, and unsorted microbiota, we used the PICRUSt pipeline (Langille et al, 2013) with the Greengenes database (gg 13_8), which yielded 7303 OTUs by closed-reference picking. For each of the three microbiota, metagenomes were predicted based on the Kyoto Encyclopedia of Genes and Genomes (KEGG, www.kegg.jp/kegg/) annotation. Average percentages for KEGG pathways were calculated for each of the three microbiota.

Statistical analyses. Microbiota alpha diversity associations with urinary oestrogens and PGE-M levels were tested with Spearman correlations. PGE-M and alpha diversity associations with casecontrol status were tested by unconditional logistic regression, with adjustment for age, body mass index (BMI), and total oestrogens. Composition of the microbiota (i.e., which microbes were found in the specimen, termed beta diversity) was compared by Bray Curtis, unweighted UniFrac and weighted UniFrac using MiRKAT (Zhao et al, 2015) and both Wald and permutation tests (with 10000 permutations) for the first three principal components (PC) of the distance matrix.

In the three sets of QC samples, mean (standard deviation) richness was 4.8 (1.8) for water, 83.3 (4.8) for Chemostat-A, and 86.4 (5.4) for Chemostat-B. Moreover, the QCs demonstrated three tight, distinct clusters on PC plots of all three beta diversity metrics (Supplementary Figure S1).

No participants or data were excluded. All statistical tests were two-sided, and $P$-values less than or equal to 0.05 were considered statistically significant.

Data availability. The microbiota $16 \mathrm{~S}$ rRNA gene sequence data and case-control status have been deposited and are available in the Sequence Read Archive (SRA) under BioProject ID PRJNA383849 (Submission ID SUB2588977).

\section{RESULTS}

The 48 postmenopausal women with breast cancer (11 in situ (stage 0$), 25$ stage 1, 10 stage 2 , two stage $3 ; 88 \%$ ER-positive) and 
48 matched control women did not differ significantly on age (median (IQR) 63 (57.5-67) vs 61 (59-64.25), $P=0.69]$ or BMI (27.92 (24.15-32.96) vs 26.78 (23.1-31.05), $P=0.30]$. Cases and controls also did not differ significantly on other characteristics relevant to breast cancer risk, as reported previously (Goedert et al, 2015). Sequencing of the 96 participants' faecal DNA revealed 1561 microbial taxa, of which 303 (8 phyla, 14 classes, 18 orders, 30 families, 83 genera, and 150 species) had a minimum relative abundance of $0.1 \%$ in at least one of the three microbiota (Supplementary Table S1). Overall richness (number of observed species) of the IgA-negative microbiota was slightly higher than the IgA-positive microbiota, and the sum of these approximated the richness of the unsorted faecal microbiota (Figure 1).

Faecal microbiota associations with case status. As reported previously (Goedert et al, 2015), the cancer cases had reduced richness and alpha diversity of their faecal microbiota. We now find that this lower richness (observed species, Figure 1) and Chaol index was significantly more marked in the IgA-positive than the IgA-negative microbiota (Table 1). Comparing lowest to highest quartiles, the relative odds of cancer were 5.39 for IgApositive observed species and 3.26 for IgA-negative observed species. For lowest-quartile $v s$ highest-quartile IgA-positive and IgA-negative alpha diversity metrics, relative odds for cancer were 5.39 and 3.16, respectively, with Chaol; 4.28 and 3.77, respectively, with PD_whole_tree; and 3.29 and 2.40, respectively, with Shannon index.

Cases and controls also differed significantly on composition of the IgA-positive microbiota (unweighted UniFrac $P=0.02$ ) and the IgA-negative microbiota fractions (unweighted UniFrac $P=0.05$, Table 1), differences that were likewise reflected in the first PC of the unweighted UniFrac distance matrix $(P=0.04$ and $P=0.03$, respectively, Table 1 and Supplementary Figure S2). The altered composition (as estimated by beta diversity) in cancer cases was of similar magnitude in the IgA-positive and IgA-negative microbiota $(P=0.66$, Table 1$)$, as further illustrated by quantilequantile (QQ) plots of observed and expected $P$-values revealing that both IgA-positive and IgA-negative species differed between cases and controls (Figure 2 top panels).

Across all phylogenetic levels, case-control differences did not meet Bonferroni significance $(P=0.00017)$ for any of the 303 taxa with at least $0.1 \%$ mean relative abundance. Case-control differences at $P<0.01$ were noted for carriage of three taxa in the IgA-positive and seven taxa in the IgA-negative microbiota (Table 2). Cases were more likely than controls to carry IgA-coated Betaproteobacteria Parasutterella (27/48 cases vs 14/48 controls, $P=0.007)$, particularly IgA-coated Betaproteobacteria Parasutterella excrementihominis $(27 / 48$ cases vs $11 / 48$ controls, $P=0.001)$. Case status was independently associated with Parasutterella carriage $\left(P_{\mathrm{adj}}=0.0003\right)$ and low richness (observed species $\left.P_{\text {adj }}=0.002\right)$.

Cases were less likely than controls to carry eight taxa including IgA-coated Firmicutes Clostridiales Ruminococcaceae Oscillibacter (41/48 cases vs $47 / 48$ controls, $P=0.003$ ), IgA-noncoated Bacteroidetes Alistipes indistinctus (18/48 cases vs 29/48 controls, $P=0.009)$, and six IgA-noncoated Firmicutes Clostridiales taxa including IgA-negative Ruminococcus (34/48 cases vs 46/48 controls, $P=0.001)$. With adjustment for richness, associations
A

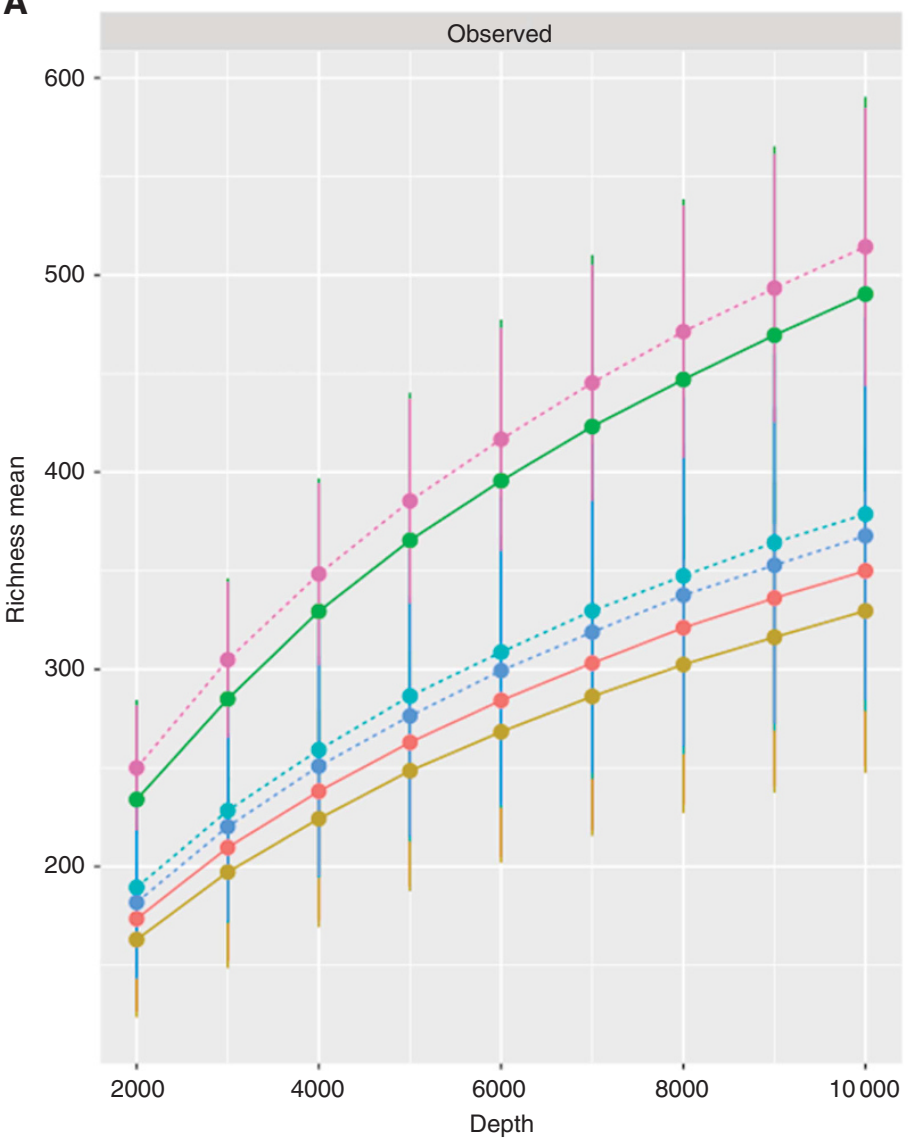

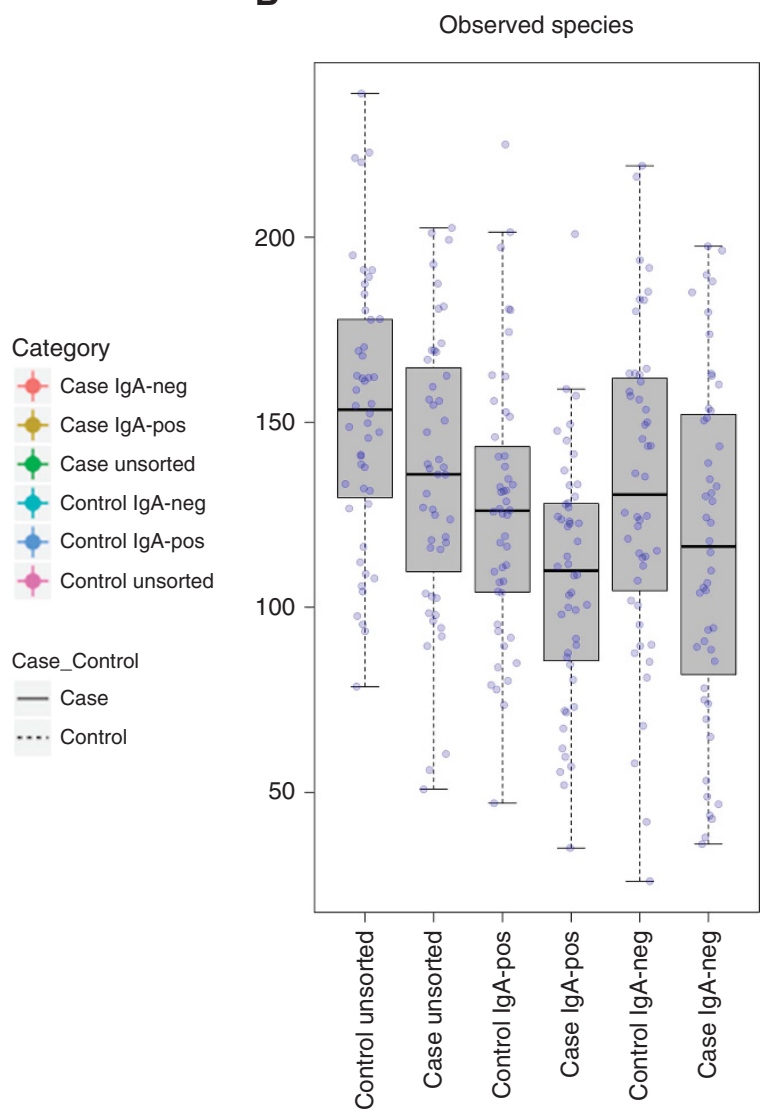

Figure 1. Richness in 96 faecal unsorted, IgA-negative and IgA-positive microbiota. (A) Rarefaction curves for observed species, for up to 10000 sequences per sample, in the unsorted, IgA-negative and IgA-positive microbiota of 48 breast cancer cases and 48 matched controls. Richness in the unsorted microbiota was approximately the sum of the IgA-negative and IgA-positive microbiotas, and in each microbiota richness was higher in controls than in cases. (B) Box plots of richness (observed species) in 48 breast cancer cases and 48 controls by microbiota. IgA $=$ immunoglobulin $A$. 
Table 1. Associations of breast cancer among postmenopausal women with microbiome metrics in faeces, before and after sorting for immunoglobulin A $(\lg A)$ staining

\begin{tabular}{|c|c|c|c|c|}
\hline & \multicolumn{4}{|c|}{$P$ value $^{a}$} \\
\hline & Unsorted & $\begin{array}{c}\lg \mathrm{A}- \\
\text { positive }\end{array}$ & $\begin{array}{c}\lg \mathrm{A}- \\
\text { negative }\end{array}$ & $\begin{array}{c}\text { IgA-positive } \\
\text { vs lgA- } \\
\text { negative }\end{array}$ \\
\hline \multicolumn{5}{|c|}{ Richness/alpha diversity metric } \\
\hline $\begin{array}{l}\text { Observed } \\
\text { species }\end{array}$ & $0.021^{\star}$ & $0.007^{\star}$ & $0.046^{*}$ & $0.054^{\star}$ \\
\hline Chao1 & $0.023^{*}$ & $0.007^{*}$ & $0.052^{*}$ & $0.045^{\star}$ \\
\hline PD_whole_tree & $0.039 *$ & $0.018^{*}$ & $0.027^{\star}$ & 0.480 \\
\hline Shannon & 0.103 & $0.041^{*}$ & 0.129 & 0.222 \\
\hline \multicolumn{5}{|c|}{ Beta diversity metric } \\
\hline $\begin{array}{l}\text { Unweighted } \\
\text { UniFrac }\end{array}$ & 0.060 & $0.023^{*}$ & $0.050^{*}$ & NE \\
\hline $\begin{array}{l}\text { Unweighted } \\
\text { UniFrac_PC1 }\end{array}$ & 0.066 & $0.037^{*}$ & $0.027^{\star}$ & 0.660 \\
\hline $\begin{array}{l}\text { Unweighted } \\
\text { UniFrac_PC2 }\end{array}$ & 0.370 & 0.815 & 0.828 & 0.940 \\
\hline $\begin{array}{l}\text { Unweighted } \\
\text { UniFrac_PC3 }\end{array}$ & 0.517 & 0.370 & 0.527 & $0.054^{\star}$ \\
\hline $\begin{array}{l}\text { Weighted } \\
\text { UniFrac }\end{array}$ & 0.253 & 0.197 & 0.195 & NE \\
\hline $\begin{array}{l}\text { Weighted } \\
\text { UniFrac_PC1 }\end{array}$ & 0.147 & 0.161 & 0.444 & 0.225 \\
\hline $\begin{array}{l}\text { Weighted } \\
\text { UniFrac_PC2 }\end{array}$ & 0.233 & 0.465 & 0.309 & 0.096 \\
\hline $\begin{array}{l}\text { Weighted } \\
\text { UniFrac_PC3 }\end{array}$ & 0.281 & 0.056 & $0.023^{*}$ & 0.550 \\
\hline Bray Curtis & 0.390 & 0.078 & 0.209 & NE \\
\hline Bray Curtis_PC1 & 0.080 & 0.079 & 0.137 & 0.389 \\
\hline Bray Curtis_PC2 & 0.847 & 0.471 & 0.380 & 0.745 \\
\hline Bray Curtis_PC3 & 0.137 & $0.050^{\star}$ & 0.280 & 0.316 \\
\hline \multicolumn{5}{|c|}{ 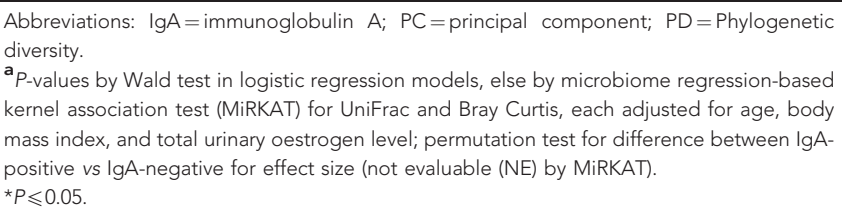 } \\
\hline
\end{tabular}

between case status and non-carriage of these IgA-noncoated taxa were attenuated $\left(P_{\mathrm{adj}}=0.02\right.$ to 0.06$)$.

Faecal microbial imputed metabolic pathway associations with case status. Abundances of eight first-level (L1), 39 second-level (L2), and 271 third-level (L3) potential metabolic pathways in the unsorted, IgA-positive and IgA-negative microbiota were derived from the PICRUSt pipeline (Langille et al, 2013) and mapped to KEGG (www.genome.jp/kegg/pathway.html). As expected, metabolic potential was generally similar among individuals and across the three microbiota fractions (Supplementary Figure S3). QQ plots for the 318 observed and expected $P$-values comparing cases $v s$ controls largely conformed to the diagonal null lines, but with a suggested deviation between cases and controls for IgA-positive L2 metabolic pathways (Figure 2 bottom panels, and Supplementary Figure S4).

Although none of the 318 metabolic pathways had Bonferronilevel statistical significance in case-control comparisons, there were five pathways (four IgA-positive, one IgA-negative) that distinguished cases from controls at $P \leqslant 0.01$ (Figure 3). Cases had higher abundance of IgA-positive 'Human Diseases; Immune System Diseases; Primary Immunodeficiency' $(P=0.003)$ and 'Immune System Diseases; Other' $(P=0.004)$ pathway genes. Adjusted for richness, cases tended to have both elevated 'Immune System Diseases' pathway genes $\left(P_{\text {adj }}=0.02\right)$ and low richness (observed species $P_{\text {adj }}=0.05$ ).
Compared to controls, cases had lower abundance IgA-positive 'Tuberculosis' pathway genes, and lower IgA-positive and IgAnegative 'Genetic Information Processing' pathway genes $(P \leqslant 0.01$ for all, Figure 3). Adjusted for richness, these pathway associations were attenuated ( $P_{\mathrm{adj}}=0.02$ to 0.12 ); and richness, adjusted for pathways, did not independently distinguish case status (observed species $P_{\text {adj }}>0.08$ ).

Prostaglandin E metabolite and oestrogen associations with microbiota alpha diversity and case status. Urinary PGE-M, oestrogen, EMs, and EM ratio distributions in cancer cases and controls are presented in Supplementary Table S2. PGE-M levels determined by the two laboratories were modestly correlated with each other in the cases (Rho 0.29, $P=0.04$ ), but not in the controls (Rho $-0.11, P=0.47$ ). BMI was positively correlated with PGE-M level in lab 2 (LC/MS method, Spearman Rho 0.26, $P=0.01$ ) but not in lab 1 (RIA method, Rho 0.04, $P=0.70$ ). Compared to controls, cases' median PGE-M values were increased $(+7.5 \%$ in lab $1,+26.6 \%$ in lab 2), but neither of these associations with case status was statistically significant (adjusted for age and BMI, $P>0.41)$. PGE-M levels were not associated with oestrogen levels (oestrone $P>0.27$; estradiol $P>0.25$; oestrone plus estradiol plus EM $P>0.129)$ nor with IgA-positive or IgA-negative microbiota alpha diversity $(P>0.20$, Table 3$)$.

Levels of parent oestrogens (oestrone and estradiol) and EMs in the major metabolic pathways $(2-\mathrm{OH}, 16-\mathrm{OH}$, and $4-\mathrm{OH})$ were all non-significantly higher in cases than in controls (Supplementary Table S2). In contrast, distributions of EM/parent oestrogen ratios (potentially reflecting differences in oestrogen metabolism) were essentially identical in cases and controls (Supplementary Table S2). As in our previous report (Goedert et al, 2015), we again found that the sum of oestrogens and EM levels was modestly correlated with faecal microbiota alpha diversity (Spearman Rho 0.18, $P=0.07$ for unsorted PD_whole_tree). We now find that this correlation was largely driven by the IgA-negative microbiota in the control women (Rho 0.36, $P=0.012$, Table 3 and Supplementary Table S3).

\section{DISCUSSION}

This study found that breast cancer in postmenopausal women was associated with significant, but differing, associations with both the immune-recognised (IgA-positive) and -unrecognised (IgA-negative) gut microbiota. In their IgA-positive microbiota, cases had increased abundance of Immune System Diseases metabolic pathways but low alpha diversity. The cases' IgA-negative microbiota also had low alpha diversity. However, unlike in the matched controls and in other healthy populations (Flores et al, 2012a; Fuhrman et al, 2014), the cancer cases' IgA-negative microbiota alpha diversity was not correlated with their oestrogen levels. These findings suggest that breast cancer risk may be influenced through enterohepatic cycling of oestrogens (Plottel and Blaser, 2011) by the IgA-negative microbiota and also through immune-mediated pathways by the IgA-positive microbiota.

Inflammation markers. Chronic inflammation has been noted to increase risk for several types of cancer (Vogtmann and Goedert, 2016). Consistent with this, elevated C-reactive protein in serum has been associated with increased risk for postmenopausal breast cancer, at least among overweight and obese women (Hong et al, 2013; Gunter et al, 2015). Extending this theme, a potential marker of inflammation, PGE-M, was reported to be modestly elevated prior to postmenopausal breast cancer diagnosis in three previous studies (Kim et al, 2013, 2017; Cui et al, 2014). In the current study, which was smaller than the previous studies and crosssectional rather than prospective, PGE-M was not significantly higher in cases than in controls. We did find that higher BMI was 
A

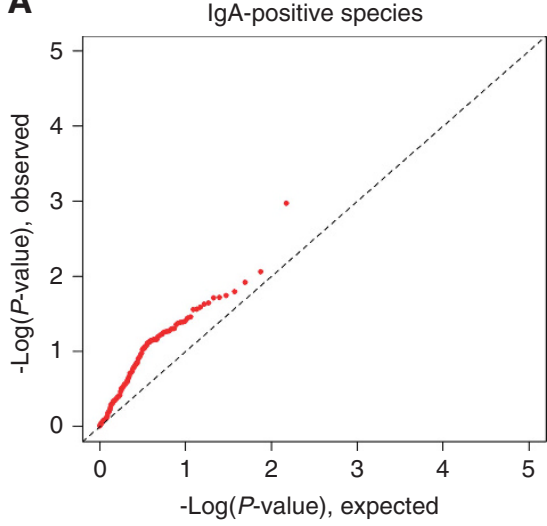

B

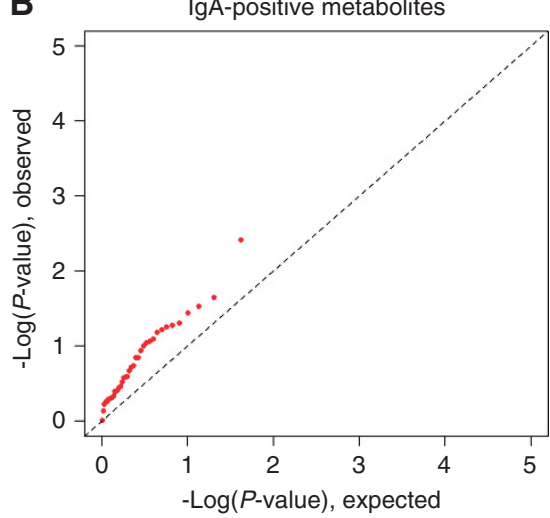

IgA-negative species

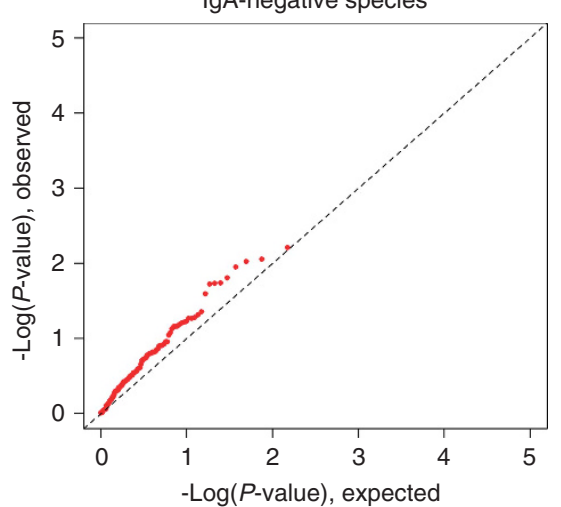

IgA-negative metabolites

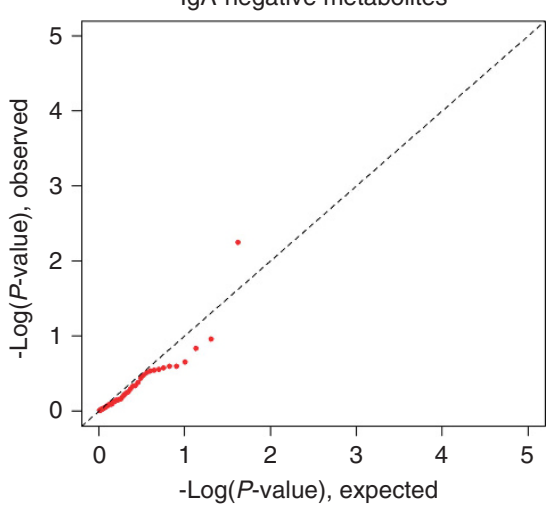

Figure 2. Beta diversity comparisons of the faecal IgA-negative and IgA-positive microbiota in postmenopausal breast cancer cases vs matched control women. (A) Distributions of observed (Y-axes) and expected (X-axes) $-\log _{10} P$-values comparing postmenopausal breast cancer cases to matched control women on detection of IgA-coated and IgA-noncoated species-level taxa. (B) Distributions of observed ( $Y$-axes) and expected (Xaxes) $-\log _{10} P$-values comparing postmenopausal breast cancer cases to matched control women on detection of IgA-coated and IgA-noncoated microbial imputed metagenomics functional pathways. $\lg \mathrm{A}=$ immunoglobulin $\mathrm{A}$.

\section{Table 2. IgA-coated and IgA-noncoated taxa potentially associated with postmenopausal breast cancer}

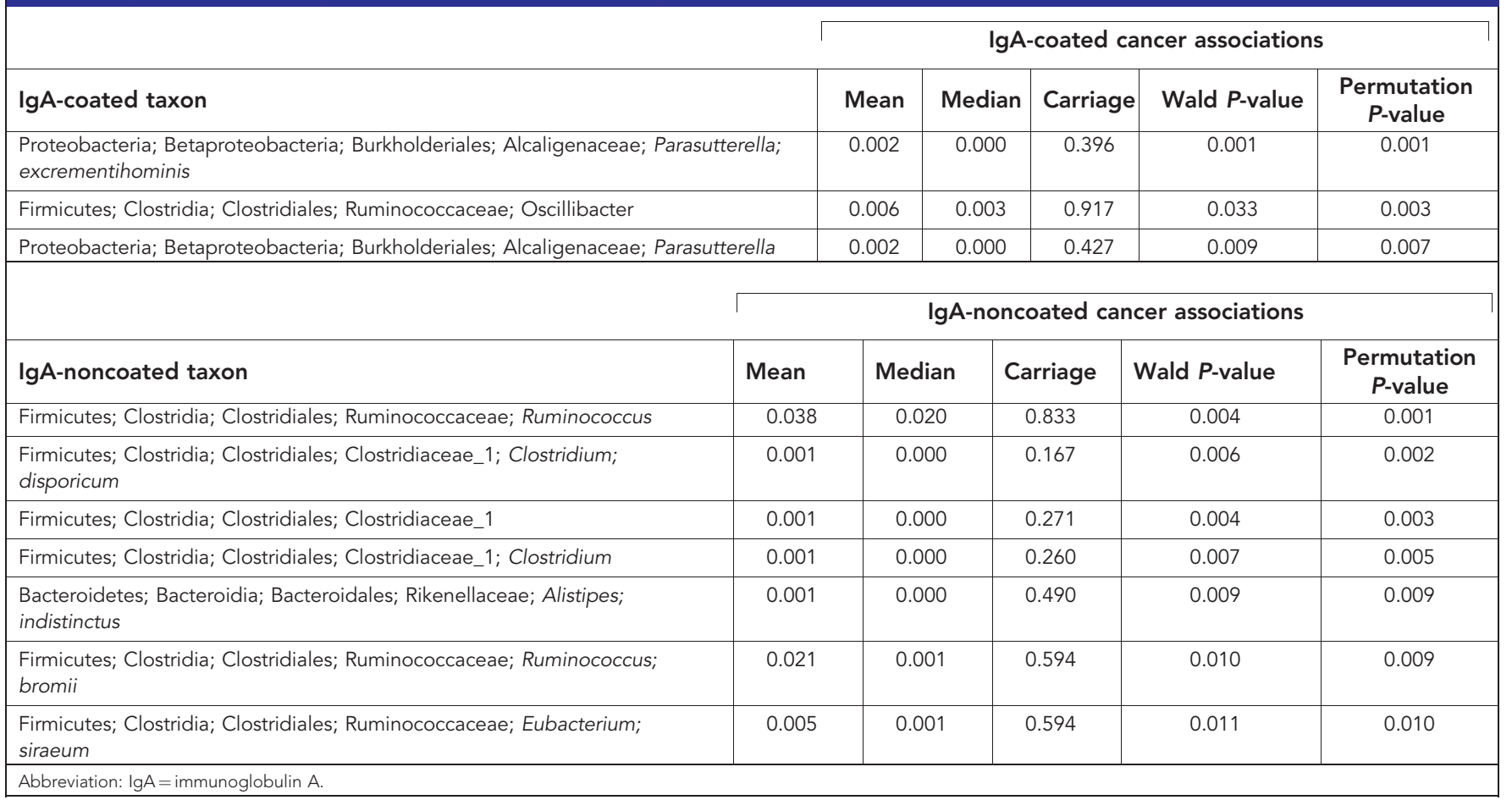



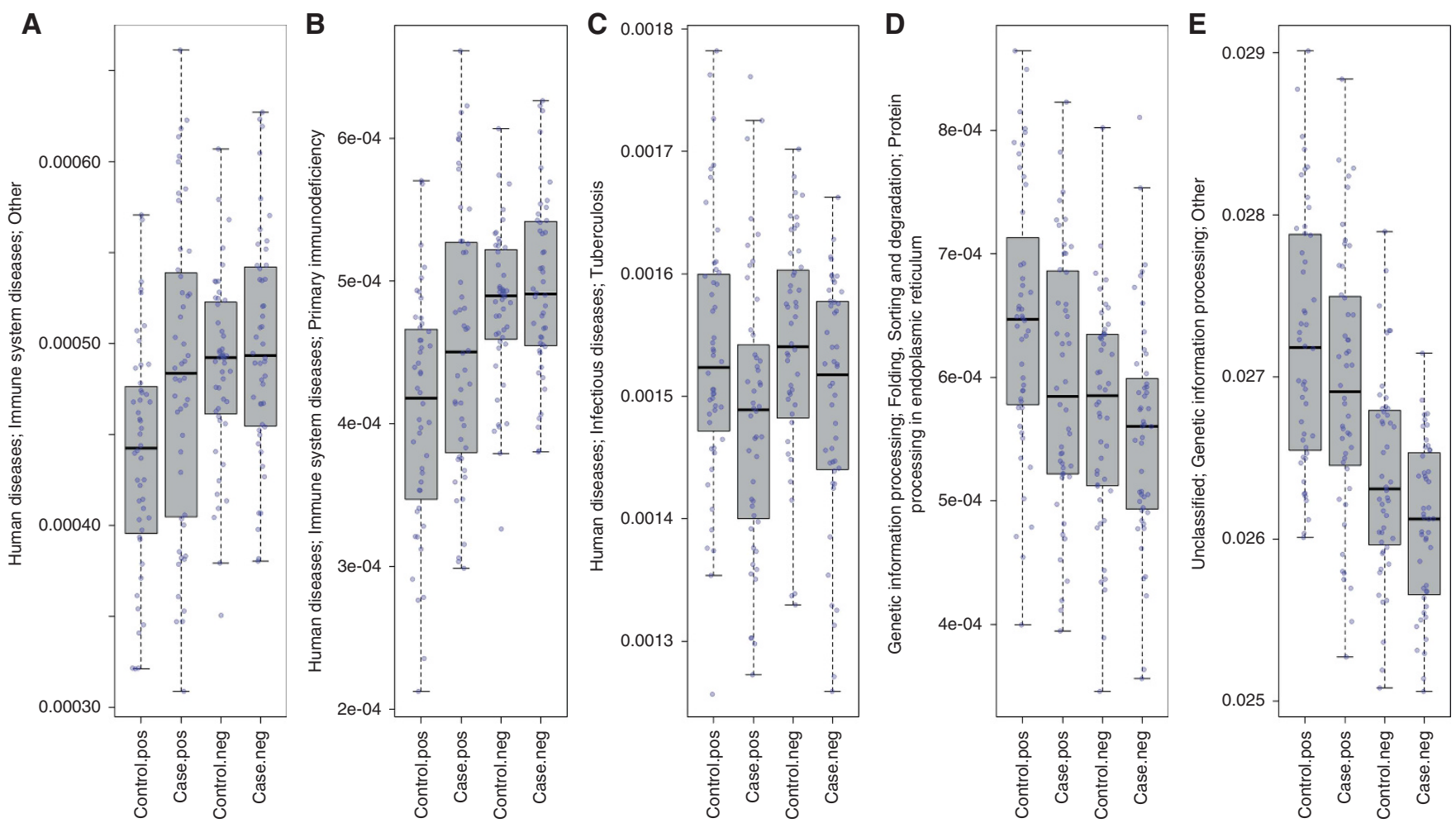

Figure 3. Relative abundances of four IgA-positive and one IgA-negative faecal microbial imputed metagenomics pathways that distinguished cases from controls at $P \leqslant 0.01$ by Wald test. (A, B) Immunoglobulin A (IgA)-positive 'Immune System Diseases; Other' and 'Primary Immunodeficiency' pathway genes were more abundant in cases than in controls. (C, D) IgA-positive 'Tuberculosis' and 'Genetic Information Processing' pathway genes were less abundant in cases. (E) IgA-negative 'Genetic Information Processing' pathway genes were less abundant in cases than in controls.

associated with higher PGE-M as determined by LC/MS but not by EIA (Morris et al, 2013; Cui et al, 2014), and we did not replicate a reported correlation between urine PGE-M and urine oestrone (E1) levels (Kim et al, 2017).

Immune-recognised gut microbes. Because immunity and inflammation may play a subtle, perhaps indirect, role in breast cancer risk, our novel approach investigated the possibility that breast cancer might be associated with immune-recognised gut microbes. Secretory IgA is produced by the gut mucosa and, when bound to certain resident microbes, reduces their fitness and mitigates the host's innate immune response against them (Peterson et al, 2007). These effects may be mediated by particular IgA-targeted epitopes, such as bacterial serine hydroxymethyltransferase (Okai et al, 2017). Commensal and especially pathogenic IgA-positive bacteria in the gut are reported to contribute to Kwashiorkor and inflammatory bowel disease, respectively (Palm et al, 2014; Kau et al, 2015). Perhaps the IgApositive gut microbiota associations that we found with cancer reflect differences in innate immunity and metabolism. Our observation that women with breast cancer had, in their IgApositive microbiota, increased Immune System Diseases and decreased Tuberculosis metabolic pathways would support this hypothesis. For example, Tuberculosis pathway genes regulate immune recognition and competition with the host for metabolism and nutrients (Warner, 2014). Thus, decreased abundance of 'tuberculosis' genes and increased abundance of 'immunodeficiency' genes in the IgA-positive gut microbiota might reflect robust albeit altered immune pressure in the cancer cases. It is unknown whether this contributes to or is a consequence of cancer, as described for inflammatory bowel disease and murine colitis (Ray and Dittel, 2015; Okai et al, 2017).
The IgA-negative (i.e., noncoated, unrecognised) microbiota could also contribute to cancer risk. The IgA-negative microbiota in our specimens was diverse and metabolically active, as shown by the direct correlation of the controls' IgA-negative alpha diversity with systemic levels of oestrone, estradiol, and their many metabolites. This suggests that the IgA-negative microbial population may be a major contributor to enterohepatic cycling of oestrogens and perhaps other bile salts (Plottel and Blaser, 2011; Dawson and Karpen, 2015). Keystone contributors to these functions are largely unknown, although investigation of species within the family Ruminococcaceae may be fruitful, as these have been associated with levels of oestrogens, functional activity of faecal $\beta$-glucuronidase, and metabolism of dietary resistant starches (Walker et al, 2011; Ze et al, 2012; Flores et al, 2012a, b). Cancer cases in the current study were lacking IgA-negative Ruminococcaceae and other Clostridiales taxa.

In both IgA-positive and IgA-negative microbiota, cases had reduced 'Genetic Information Processing' gene abundances. KEGG (www.kegg.jp/kegg/) summarises that this DNA-replication pathway is a complex network of proteins and enzymes. In prokaryotes this pathway includes a pol III core DNA polymerase, a beta sliding clamp, a gamma delta complex clamp loader, a DNA primase (DnaG), removal of an RNA primer by an RNase $\mathrm{H}$ or by the 5-to-3 exonuclease activity of DNA pol I, and finally a DNA ligase to join the fragments. If reduced 'Genetic Information Processing' microbial genes in breast cancer cases is independently validated, deeper investigation will be required to understand the functional significance, which might include reduced microbial replication and metabolism with cancer.

We did not observe associations of the IgA-negative or the IgApositive microbiota with altered ratios of parent oestrogens to their metabolites, thus providing no support to the hypothesis that the 
Table 3. Spearman rank-order correlations of prostaglandin $E$ metabolite (PGE-M1 in laboratory 1, PGE-M2 in laboratory 2), total urinary oestrogens, and the PD_whole_tree estimate of faecal microbiota alpha diversity, by case-control status

\begin{tabular}{|c|c|c|c|c|c|c|}
\hline \multirow{2}{*}{$\begin{array}{l}\text { Assay pair } \\
\text { Oestrogens \& } \\
\text { PGE-M }\end{array}$} & \multicolumn{2}{|c|}{ Controls } & \multicolumn{2}{|c|}{ Cases } & \multicolumn{2}{|c|}{ All participants } \\
\hline & rho & $\begin{array}{c}P- \\
\text { value }\end{array}$ & rho & $\begin{array}{c}P- \\
\text { value }\end{array}$ & rho & $\begin{array}{c}P- \\
\text { value }\end{array}$ \\
\hline $\begin{array}{l}\text { Oestrogens vs } \\
\text { PGE-M1 }\end{array}$ & 0.325 & $0.024^{*}$ & 0.004 & 0.976 & 0.156 & 0.129 \\
\hline $\begin{array}{l}\text { Oestrogens vs } \\
\text { PGE-M2 }\end{array}$ & 0.111 & 0.461 & -0.332 & $0.021 *$ & -0.108 & 0.298 \\
\hline $\begin{array}{l}\text { PGE-M1 vs PGE- } \\
\text { M2 }\end{array}$ & -0.109 & 0.469 & 0.294 & $0.043^{*}$ & 0.108 & 0.301 \\
\hline \multicolumn{7}{|l|}{$\begin{array}{l}\text { Unsorted } \\
\text { microbiota }\end{array}$} \\
\hline $\begin{array}{l}\text { Oestrogens vs } \\
\text { PD_whole_tree }\end{array}$ & 0.209 & 0.154 & 0.201 & 0.175 & 0.184 & 0.074 \\
\hline & 0.108 & 0.464 & -0.141 & 0.343 & -0.027 & 0.798 \\
\hline $\begin{array}{l}\text { PGE-M2 vs } \\
\text { PD_whole_tree }\end{array}$ & -0.056 & 0.713 & -0.181 & 0.225 & -0.128 & 0.222 \\
\hline \multicolumn{7}{|l|}{$\begin{array}{l}\text { IgA-positive } \\
\text { microbiota }\end{array}$} \\
\hline $\begin{array}{l}\text { Oestrogens vs } \\
\text { PD_whole_tree }\end{array}$ & 0.125 & 0.398 & 0.236 & 0.107 & 0.152 & 0.140 \\
\hline $\begin{array}{l}\text { PGE-M1 vs } \\
\text { PD_whole_tree }\end{array}$ & 0.090 & 0.545 & -0.061 & 0.682 & 0.001 & 0.992 \\
\hline $\begin{array}{l}\text { PGE-M2 vs } \\
\text { PD_whole_tree }\end{array}$ & -0.157 & 0.297 & -0.109 & 0.462 & -0.132 & 0.204 \\
\hline \multicolumn{7}{|l|}{$\begin{array}{l}\text { IgA-negative } \\
\text { microbiota }\end{array}$} \\
\hline $\begin{array}{l}\text { Oestrogens vs } \\
\text { PD_whole_tree }\end{array}$ & 0.363 & $0.012^{\star}$ & 0.221 & 0.132 & 0.251 & $0.014^{\star}$ \\
\hline $\begin{array}{l}\text { PGE-M1 vs } \\
\text { PD_whole_tree }\end{array}$ & 0.069 & 0.643 & -0.033 & 0.822 & -0.001 & 0.993 \\
\hline $\begin{array}{l}\text { PGE-M2 vs } \\
\text { PD_whole_tree }\end{array}$ & -0.110 & 0.467 & -0.092 & 0.532 & -0.113 & 0.278 \\
\hline
\end{tabular}

Abbreviations: IgA = immunoglobulin $\mathrm{A} ; \mathrm{PD}=$ phylogenetic diversity; $\mathrm{PGE}=$ prostaglandin E. ${ }^{*} P \leqslant 0.05$.

microbiota substantially metabolises oestrogens (Fuhrman et al, 2014). Rather, other factors, such host differences in hepatic enzyme activities, may underlie associations of metabolite/parent oestrogen ratios with breast cancer risk (Sampson et al, 2017).

Strengths and weaknesses. This study had noteworthy strengths, including a well-defined population base, unbiased ascertainment of breast cancer cases and closely matched controls, state-of-the-art laboratory and statistical analysis methods, and demonstration of high reproducibility in 33 carefully curated QC samples. This study also had important limitations, including small sample size that precluded detection of weak associations, analysis of potential metabolic pathways that were derived by imputation but not validated with an independent method, and cross-sectional design such that reverse causality (i.e., cancer causing the observed differences) cannot be excluded. The pathway data and associations must be interpreted with caution. The design limitation was addressed by recruiting only asymptomatic cases whose tumours were detected by screening mammography and by collecting their specimens prior to surgical or systemic therapy.

\section{CONCLUSION}

In both the IgA-positive and the IgA-negative faecal microbiota, differences in alpha diversity, composition, imputed metabolic pathways, and associations with urinary oestrogen levels were observed between postmenopausal women with untreated breast cancer and matched control women. Examining these associations in prospective cohort studies with longitudinally collected prediagnostic specimens may provide insights on gut microbial contributions to breast cancer and potential targets for intervention.

\section{ACKNOWLEDGEMENTS}

This study was supported in part by the National Cancer Institute Intramural Research Program (ZIA CP 010214).

\section{CONFLICT OF INTEREST}

The authors declare no conflict of interest.

\section{AUTHOR CONTRIBUTIONS}

JJG conceived the project, recruited the collaborators, and drafted the manuscript. NWP designed and supervised the faecal microbiota experiments. $\mathrm{AB}$ performed the microbiome experiments and initial microbiome data processing. XX designed and supervised the oestrogen experiments. IO, MF, and GLM designed and supervised the PGE-M experiments in their respective laboratories. RS contributed the Chemostat QC specimens and led the QC analyses. GSJ selected the specimens, managed their distribution, and performed initial analyses. JR contributed to the project design and to interpretation of the data. YW performed the informatics, and generated the operational taxonomic unit, relative abundance, and metabolic pathway tables. XH performed the final statistical analyses and generated the tables and most of the figures. JS supervised the informatics and statistical analyses. HSF organised, managed, and supervised the field work. All authors contributed to the final manuscript.

\section{REFERENCES}

Adlercreutz H, Martin F (1980) Biliary excretion and intestinal metabolism of progesterone and estrogens in man. J Steroid Biochem 13(2): 231-244.

Bardia A, Olson JE, Vachon CM, Lazovich D, Vierkant RA, Wang AH, Limburg PJ, Anderson KE, Cerhan JR (2011) Effect of aspirin and other NSAIDs on postmenopausal breast cancer incidence by hormone receptor status: results from a prospective cohort study. Breast Cancer Res Treat 126(1): 149-155.

Boulangé CL, Neves AL, Chilloux J, Nicholson JK, Dumas ME (2016) Impact of the gut microbiota on inflammation, obesity, and metabolic disease. Genome Med 8(1): 42.

Bowers LW, Brenner AJ, Hursting SD, Tekmal RR, deGraffenried LA (2015) Obesity-associated systemic interleukin- 6 promotes pre-adipocyte aromatase expression via increased breast cancer cell prostaglandin E2 production. Breast Cancer Res Treat 149(1): 49-57.

Bowers LW, Maximo IX, Brenner AJ, Beeram M, Hursting SD, Price RS, Tekmal RR, Jolly CA, deGraffenried LA (2014) NSAID use reduces breast cancer recurrence in overweight and obese women: role of prostaglandinaromatase interactions. Cancer Res 74(16): 4446-4457.

Brown KA, Simpson ER (2012) Obesity and breast cancer: mechanisms and therapeutic implications. Front Biosci (Elite Ed) 4: 2515-2524.

Callahan BJ, McMurdie PJ, Rosen MJ, Han AW, Johnson AJ, Holmes SP (2016) DADA2: high-resolution sample inference from Illumina amplicon data. Nat Methods 13(7): 581-583.

Cui Y, Shu XO, Gao YT, Cai Q, Ji BT, Li HL, Rothman N, Wu J, Yang G, Xiang YB, Zheng W (2014) Urinary prostaglandin E2 metabolite and breast cancer risk. Cancer Epidemiol Biomarkers Prev 23(12): 2866-2873. Dawson PA, Karpen SJ (2015) Intestinal transport and metabolism of bile acids. J Lipid Res 56(6): 1085-1099. 
de Pedro M, Baeza S, Escudero MT, Dierssen-Sotos T, Gómez-Acebo I, Pollán M, Llorca J (2015) Effect of COX-2 inhibitors and other nonsteroidal inflammatory drugs on breast cancer risk: a meta-analysis. Breast Cancer Res Treat 149(2): 525-536.

Feigelson HS, Bischoff K, Ardini MA, Ravel J, Gail MH, Flores R, Goedert JJ (2014) Feasibility of self-collection of fecal specimens by randomly sampled women for health-related studies of the gut microbiome. $B M C$ Res Notes 7: 204.

Flores R, Shi J, Fuhrman B, Xu X, Veenstra TD, Gail MH, Gajer P, Ravel J, Goedert JJ (2012a) Fecal microbial determinants of fecal and systemic estrogens and estrogen metabolites: a cross-sectional study. J Transl Med 10: 253.

Flores R, Shi J, Gail MH, Gajer P, Ravel J, Goedert JJ (2012b) Association of fecal microbial diversity and taxonomy with selected enzymatic functions. PLoS One 7(6): e39745.

Fuhrman BJ, Feigelson HS, Flores R, Gail MH, Xu X, Ravel J, Goedert JJ (2014) Associations of the fecal microbiome with urinary estrogens and estrogen metabolites in postmenopausal women. J Clin Endocrinol Metab 99(12): 4632-4640.

Gierach GL, Lacey JV, Schatzkin A, Leitzmann MF, Richesson D, Hollenbeck AR, Brinton LA (2008) Nonsteroidal anti-inflammatory drugs and breast cancer risk in the National Institutes of Health-AARP Diet and Health Study. Breast Cancer Res 10(2): R38.

Goedert JJ, Jones G, Hua X, Xu X, Yu G, Flores R, Falk RT, Gail MH, Shi J, Ravel J, Feigelson HS (2015) Investigation of the association between the fecal microbiota and breast cancer in postmenopausal women: a population-based case-control pilot study. J Natl Cancer Inst 107(8): (pii): djv147.

Gunter MJ, Wang T, Cushman M, Xue X, Wassertheil-Smoller S, Strickler HD, Rohan TE, Manson JE, McTiernan A, Kaplan RC, Scherer PE, Chlebowski RT, Snetselaar L, Wang D, Ho GY (2015) Circulating adipokines and inflammatory markers and postmenopausal breast cancer risk. J Natl Cancer Inst; doi: 10.1093/jnci/djv169.

Hong T, Liu A, Cai D, Zhang Y, Hua D, Hang X, Wu X (2013) Preoperative serum C-reactive protein levels and early breast cancer by BMI and menopausal status. Cancer Invest 31(4): 279-285.

Kau AL, Planer JD, Liu J, Rao S, Yatsunenko T, Trehan I, Manary MJ, Liu TC, Stappenbeck TS, Maleta KM, Ashorn P, Dewey KG, Houpt ER, Hsieh CS, Gordon JI (2015) Functional characterization of IgA-targeted bacterial taxa from undernourished Malawian children that produce dietdependent enteropathy. Sci Transl Med 7(276): $276 \mathrm{ra} 24$.

Key T, Appleby P, Barnes I, Reeves G. Group EHaBCC (2002) Endogenous sex hormones and breast cancer in postmenopausal women: reanalysis of nine prospective studies. J Natl Cancer Inst 94(8): 606-616.

Key TJ, Appleby PN, Reeves GK, Roddam A, Dorgan JF, Longcope C, Stanczyk FZ, Stephenson HE, Falk RT, Miller R, Schatzkin A, Allen DS, Fentiman IS, Wang DY, Dowsett M, Thomas HV, Hankinson SE, Toniolo P, Akhmedkhanov A, Koenig K, Shore RE, Zeleniuch-Jacquotte A, Berrino F, Muti P, Micheli A, Krogh V, Sieri S, Pala V, Venturelli E, Secreto G, Barrett-Connor E, Laughlin GA, Kabuto M, Akiba S, Stevens RG, Neriishi K, Land CE, Cauley JA, Kuller LH, Cummings SR, Helzlsouer KJ, Alberg AJ, Bush TL, Comstock GW, Gordon GB, Miller SR, Group EHBCC (2003) Body mass index, serum sex hormones, and breast cancer risk in postmenopausal women. J Natl Cancer Inst 95(16): 1218-1226.

Kim S, Campbell J, Yoo W, Taylor JA, Sandler DP (2017) Systemic levels of estrogens and PGE2 synthesis in relation to postmenopausal breast cancer risk. Cancer Epidemiol Biomarkers Prev 26(3): 383-388.

Kim S, Taylor JA, Milne GL, Sandler DP (2013) Association between urinary prostaglandin E2 metabolite and breast cancer risk: a prospective, case-cohort study of postmenopausal women. Cancer Prev Res (Phila) 6(6): 511-518.

Kwa M, Plottel CS, Blaser MJ, Adams S (2016) The intestinal microbiome and estrogen receptor-positive female breast cancer. J Natl Cancer Inst; doi: 10.1093/jnci/djw029.

Langille MG, Zaneveld J, Caporaso JG, McDonald D, Knights D, Reyes JA, Clemente JC, Burkepile DE, Vega Thurber RL, Knight R, Beiko RG, Huttenhower C (2013) Predictive functional profiling of microbial communities using $16 \mathrm{~S}$ rRNA marker gene sequences. Nat Biotechnol 31(9): 814-821.

Morris PG, Zhou XK, Milne GL, Goldstein D, Hawks LC, Dang CT, Modi S, Fornier MN, Hudis CA, Dannenberg AJ (2013) Increased levels of urinary PGE-M, a biomarker of inflammation, occur in association with obesity, aging, and lung metastases in patients with breast cancer. Cancer Prev Res (Phila) 6(5): 428-436.
Okai S, Usui F, Ohta M, Mori H, Kurokawa K, Matsumoto S, Kato T, Miyauchi E, Ohno H, Shinkura R (2017) Intestinal IgA as a modulator of the gut microbiota. Gut Microbes 8(5): 486-492.

Okayasu I, Ohnishi H, Sarandi I, Shojima J, Komatsu J, Oritsu M, Sasabe M, Nanami KO, Matsuura M, Azumi J, Ito S, Fujiwara M (2014) Significant increase of prostaglandin E-major urinary metabolite in male smokers: a screening study of age and gender differences using a simple radioimmunoassay. J Clin Lab Anal 28(1): 32-41.

Palm NW, de Zoete MR, Cullen TW, Barry NA, Stefanowski J, Hao L, Degnan PH, Hu J, Peter I, Zhang W, Ruggiero E, Cho JH, Goodman AL, Flavell RA (2014) Immunoglobulin A coating identifies colitogenic bacteria in inflammatory bowel disease. Cell 158(5): 1000-1010.

Peterson DA, McNulty NP, Guruge JL, Gordon JI (2007) IgA response to symbiotic bacteria as a mediator of gut homeostasis. Cell Host Microbe 2(5): 328-339.

Plottel CS, Blaser MJ (2011) Microbiome and malignancy. Cell Host Microbe 10(4): 324-335.

Ray A, Dittel BN (2015) Interrelatedness between dysbiosis in the gut microbiota due to immunodeficiency and disease penetrance of colitis. Immunology 146(3): 359-368.

Sampson JN, Falk RT, Schairer C, Moore SC, Fuhrman BJ, Dallal CM, Bauer DC, Dorgan JF, Shu XO, Zheng W, Brinton LA, Gail MH, Ziegler RG, Xu X, Hoover RN, Gierach GL (2017) Association of estrogen metabolism with breast cancer risk in different cohorts of postmenopausal women. Cancer Res 77(4): 918-925.

Santiago-Rodriguez TM, Ly M, Daigneault MC, Brown IH, McDonald JA, Bonilla N, Vercoe EA, Pride DT (2015) Chemostat culture systems support diverse bacteriophage communities from human feces. Microbiome 3: 58.

Silva MT, Galvao TF, Zimmerman IR, Pereira MG, Lopes LC (2012) Non-aspirin non-steroidal anti-inflammatory drugs for the primary chemoprevention of non-gastrointestinal cancer: summary of evidence. Curr Pharm Des 18(26): 4047-4070.

Subbaramaiah K, Morris PG, Zhou XK, Morrow M, Du B, Giri D, Kopelovich L, Hudis CA, Dannenberg AJ (2012) Increased levels of COX2 and prostaglandin E2 contribute to elevated aromatase expression in inflamed breast tissue of obese women. Cancer Discov 2(4): 356-365.

Tamimi RM, Spiegelman D, Smith-Warner SA, Wang M, Pazaris M, Willett WC, Eliassen AH, Hunter DJ (2016) Population attributable risk of modifiable and nonmodifiable breast cancer risk factors in postmenopausal breast cancer. Am J Epidemiol 184(12): 884-893.

Tikkanen MJ, Adlercreutz H, Pulkkinen MO (1973) Effects of antibiotics on oestrogen metabolism. Br Med J 2(5862): 369.

Vogtmann E, Goedert JJ (2016) Epidemiologic studies of the human microbiome and cancer. Br J Cancer 114(3): 237-242.

Walker AW, Ince J, Duncan SH, Webster LM, Holtrop G, Ze X, Brown D, Stares MD, Scott P, Bergerat A, Louis P, McIntosh F, Johnstone AM, Lobley GE, Parkhill J, Flint HJ (2011) Dominant and diet-responsive groups of bacteria within the human colonic microbiota. ISME J 5(2): 220-230.

Warner DF (2014) Mycobacterium tuberculosis metabolism. Cold Spring Harb Perspect Med; doi:10.1101/cshperspect.a021121.

Yiannakopoulou ECh (2015) Aspirin and NSAIDs for breast cancer chemoprevention. Eur J Cancer Prev 24(5): 416-421.

Ze X, Duncan SH, Louis P, Flint HJ (2012) Ruminococcus bromii is a keystone species for the degradation of resistant starch in the human colon. ISME J 6(8): 1535-1543.

Zhao N, Chen J, Carroll IM, Ringel-Kulka T, Epstein MP, Zhou H, Zhou JJ, Ringel Y, Li H, Wu MC (2015) Testing in microbiome-profiling studies with MiRKAT, the microbiome regression-based kernel association test. Am J Hum Genet 96(5): 797-807.

Zhong S, Chen L, Zhang X, Yu D, Tang J, Zhao J (2015) Aspirin use and risk of breast cancer: systematic review and meta-analysis of observational studies. Cancer Epidemiol Biomarkers Prev 24(11): 1645-1655.

This work is published under the standard license to publish agreement. After 12 months the work will become freely available and the license terms will switch to a Creative Commons AttributionNonCommercial-Share Alike 4.0 Unported License. 